\title{
A REVIEW ON DATA MINING AND BIGDATA
}

\author{
Aarepu Lakshman \\ Research Scholar, Shri Jagdishprasad Jhabarmal Tibrewala University, \\ Rajasthan, India \\ Dr. B.M.G. Prasad \\ Professor, Department of CSE and Dean, \\ Holy Mary Institute of Technology and Science Hyderabad, Telangana \\ Dr. Yogesh Kumar Sharma \\ Associate Professor \& HOD Department of CSE \& IT, \\ Shri Jagdishprasad Jhabarmal Tibrewala University, Jhunjhunu, Rajasthan
}

\begin{abstract}
The recent years have seen an exponential growth of data generation this enormous amount of data has brought new kind of problem. The existing data mining techniques are unable to process the Big Data or they are not efficient in handling it. The major problems appeared with the Big Data are storage and processing, and how big data process efficiently in order to this high computation platforms required. To extract valuable information from big data appropriate algorithms required. To deeply understand this issue and to find recommendations in this paper taking review from previous studies. First part of review explores how data analysis done with data mining algorithms and its limitations. Second part of review explores data analysis with big data and with different algorithms and framework.
\end{abstract}

Keywords: Data Mining, Big Data, Data Analysis

Cite this Article: Aarepu Lakshman, Dr. B.M.G. Prasad and Dr. Yogesh Kumar Sharma, A Review on Data Mining and Bigdata, International Journal of Computer Engineering and Technology, 10(1), 2019, pp. 117-123.

http://iaeme.com/Home/issue/IJCET?Volume=10\&Issue=1

\section{INTRODUCTION}

We live in a world which is excessively dependent and driven by data. There has been an explosion of data in recent years. 90 percent of the data in the world has been generated in the last two years; more data is generated in the past two years than the entire history of the humankind. Data is growing exponentially, by the 2020 it is expected that every person on the will generate 1.7 MB of data every second on average. The total accumulated data 4.4 Zettabytes presently will go up to 44 Zetta bytes, or 44 trillion Gigabytes by 2020 . By then we will have over 6.1 billion smartphone users, 50 billion devices connected to internet and out 
of all the accumulated data so far one third of it will be stored on cloud. So far out of all the accumulated data only $0.5 \%$ is actually analyzed and used, this shows the huge potential the of data analyzation here [1]. The IDC report [9] indicates that big data marketing in 2014 is about \$16.1 billion. Another IDC report [10] predicts that it will reach \$32.4 billion by 2017 . Reports from [11] and [12] He also pointed out that big data marketing would be $\$ 46.34$ billion and $\$ 114$ billion, respectively reach in 2018. Although the big data marketing values in these surveys and technology reports $[9,10,11,12,13,14,15]$ are different, these forecasts generally indicate that the big data field will increase rapidly in the next years will increase to come . In addition to marketing, the results of disease control and prevention [16], strategic intelligence [17] and the smart city [18], we can easily understand that big data is vital everywhere. Many researches are therefore focused on the development of efficient technologies for the analysis of Big Data. To deepen the analysis of large data, this article provides not only a systematic description of traditional large-scale data analysis, but also a detailed discussion of the differences between the framework for data analysis and large data, so that scientists or researchers can focus on big data analysis.

Data is a critical resource which exists in many forms. Big data do not have a standard definition while it denotes in several ways. The term big data is referred to describe the exponential growth of the data flow in various sectors which is too large to process using the available traditional database and software techniques. Often big data is considered to be scary, yet it is an explosion in the field of knowledge. It helps to implement various analytics, which can create an impression on the economic growth, generating events, increasing efficiency over other methods. This critical collection of data often described as threedimensional namely Volume, Velocity and Variety where some even define with Veracity (Katal, Avita, et al, 2015) [2]..

Volume: With advent of social media and smart phones, enormous volumes of data is being generated. Apart from this, a lot of businesses started collecting real time stream data which is voluminous in nature.

Velocity: Data is generated at a faster rate, which makes it even more arduous to manage.

Veracity: This is about the trustworthiness of data.

Variety: Different formats in which data is generated, structured, semi structured and unstructured. A total of 90 percent of the generated in the last two years is unstructured (Sivarajah, Uthayasankar, et al., 2017) [3][7].

\section{LITERATURE REVIEW}

Literature review described as the summary or re-organizing the related information from different sources to understand the research problem. In this review how data analysis done for big data through data mining techniques is studied.

\section{DATA ANALYSIS FOR DATA MINING}

Because data analysis in KDD is responsible for looking for hidden data schemas/rules/ information, most researchers in this area use the term data mining to describe how it perfects the "ground" in "gold nugget". [20] Are not limited to data-specific methods other technologies (eg statistical or machine learning technologies) have been used for years to analyze data In the early stages of data analysis, statistical methods were used to analyze the data to help us Understanding the situation we are facing, such as the public opinion survey or planned TV assessment Just as with statistical analysis, the specific methods of the data mining problem have also tried to understand the meaning of the data collected. 
After the problem with data mining, some domain-specific algorithms are also developed. An example is the apriori algorithm, which is one of the useful algorithms designed for the problem of association rules. Although most data mining problems are simple to define, the computing costs are quite high. To speed up the response time of a data mining operator, machine learning, metaheuristic algorithms and distributed processing are only used or combined with traditional exploration algorithms in order to provide more efficient methods of solving data mining problems. One of the known combinations can be found in, Krishna and Murty have tried to combine the genetic algorithm and the k-means to obtain better cluster results than the k-means alone.

Clustering is one of the known problems of data mining because it can be used to understand the "new" input data. The basic idea of this problem is to separate a series of nonlabel 2 input data into several groups, eg as k-means. The classification [20] is the opposite of the classification because it is based on a series of labeled input data to construct a set of classifiers (i.e. groups) which will then be used to classify the data untagged entry in the groups to which they belong. In order to solve the classification problem, the algorithm based on a decision tree, the Bayes naive classification and the machine vector support (SVM) have been widely used in recent years.

Unlike classification and classification methods that attempt to classify input data into kgroups, association rules and sequential models focus on finding "relationships" between input data. The basic idea of association rules is to find all the relations of co-occurrence between the input data. For the problem of association rules, the apriori algorithm is one of the most popular methods. However, since it is very expensive to calculate, follow-up studies have tried to use different approaches to reduce the cost of the algorithm a priori, such as the application of the genetic algorithm to this problem. In addition to examining the relationships between input data, if we also consider the sequence or time series of input data, we are talking about the problem of sequential exploration. Several apriori-like algorithms have been presented to solve it, such as the generalized sequential model and the sequential discovery of the model using equivalence classes.

The analogous situation also exists in data classification and classification studies, because the basic concept of the previous algorithms, such as the extraction of models during flight, the extraction of partial models in different phases and reducing the number of times that the complete dataset is used scanned to be presented to improve the performance of these data mining algorithms. Since some data mining problems are very difficult to solve or the solution space is very large, several recent surveys have attempted to use the meta system algorithm as an algorithm to obtain the approximate solution within a reasonable time to send

Table 1 Data analysis methods for Data Mining

\begin{tabular}{|c|c|}
\hline Problem & Method \\
\hline Clustering & BIRCH,DBSCAN, Incremental DBSCAN, RKM, TKM \\
\hline Classification & SLIQ, TLAESA, FastNN, SFFS, GPU-based SVM \\
\hline Association Rules & CLOSET, FP-TREE, CHARM, MAFIA, FAST \\
\hline Sequential patterns & SPADE, CloSpan, PrefixSpan, SPAM, ISE. \\
\hline
\end{tabular}




\section{PROBLEM STATEMENT}

The traditional database systems are not built to handle the kind of huge amounts of data we are experiencing in the recent years. It is also very expensive to increase the processing power on these systems. Also the traditional systems can only process structured data. The major part of the data generated in last two years is unstructured data. The existing system works on a single server, which makes it difficult and costly to grow it vertically. Numerous research results on data analysis [20] show possible solutions for solving the dilemmas of data mining algorithms. This means that open questions about the analysis of literature data [2] can usually help us to easily find possible solutions. For example, the clustering result is extremely sensitive to the initial means, which can be mitigated using different sets of initial means. According to our observations, most data analysis methods have limitations for Big Data that can be described as follows:

- Unscalability and centralization

- Non-dynamic

- Uniform data structure

Because the traditional data analysis methods are not designed for large-scale and complex data, they are almost impossible to be capable of analyzing the big data. Redesigning and changing the way the data analysis methods are designed are two critical trends for big data analysis. Several important concepts in the design of the big data analysis method will be given in the following sections.

\section{LIMITATIONS}

The main two disadvantages are:

1. Cannot store and process Big Data

2. Cannot process unstructured data

There is limit to how much one can grow this system vertically. Most of these single servers are high end or custom made, hence not cost effective.

\section{DATA ANALYTICS FOR BIG DATA}

Nowadays, the data to be analyzed is not only large, it consists of different types of data and even contains continuous data. Given that big data has the unique characteristics of "massive, large, heterogeneous, complex, unstructured, incomplete, noisy, and inaccurate mass," this could change the approach to statistics and data analysis. Although it seems that Big Data allows us to collect more data to find more useful information, the truth is that more data does not necessarily mean more useful information. It may contain more ambiguous or abnormal data. For example, a user can have multiple accounts or an account can be used by multiple users, which can reduce the accuracy of the results of the scan. As a result, there are several new data analysis issues, such as privacy, security, storage, fault tolerance, and data quality.

Based on the results of recent Big Data analytics, Nolan's growth model is still in its infancy, comparable to the themes of cloud computing, cloud computing, and cloud-based research. Internet of Things and Smart Network. Indeed, several studies have simply tried to apply traditional solutions to new problems / platforms / environments. For example, several studies have used k-means as an example to analyze large data, but few have applied data mining algorithms and advanced algorithms for machine learning to large data analysis. This explains why the performance of large data analytics can be improved with data mining algorithms and metaeistic algorithms presented in recent years. 
The compression, sampling, or even platform technologies presented in recent years can also be used to improve the performance of the large data analysis system. Although these research topics still raise several outstanding issues, these situations also show that anything is possible in these studies.

In below table discussed about different big data analysis frameworks and methods

Table 2 Big data analysis frameworks

\begin{tabular}{|c|c|}
\hline Big data Framework & Description \\
\hline DOT & $\begin{array}{l}\text { It is analysis framework and it has more computation resource to scale out } \\
\qquad \text { the data }\end{array}$ \\
\hline GLADE & $\begin{array}{l}\text { It is data analysis framework and it is tree based multi-level system } \\
\qquad \text { architecture }\end{array}$ \\
\hline STARFISH & This analysis framework designed for self-tuning \\
\hline ODT-MDC & This analysis framework intention to developed for privacy issues \\
\hline MRAM & $\begin{array}{l}\text { It is data analysis framework, specially it designed for mobile agent } \\
\text { technologies }\end{array}$ \\
\hline CBDMASP & This framework intended for statistical computation of big data \\
\hline SODSS & This framework for Data decision support \\
\hline BDAF & This framework support data centric architecture. \\
\hline HACE & It defines properties of big data \\
\hline HADOOP & It is a platform designed for distributed and parallel data processing \\
\hline CUDA & It is also a platform intended for parallel computing \\
\hline STORM & It is a data analysis platform designed for parallel computing \\
\hline PREGEL & This platform for large scale graph data analysis \\
\hline MLPACK & It is a rich library for of machine learning and \\
\hline MAHOUT & $\begin{array}{c}\text { It consist algorithms for clustering, classification it provides math libraries } \\
\text { from java and also linear algebra. }\end{array}$ \\
\hline MLAS & It provides different libraries of math and statistics \\
\hline RADOOP & $\begin{array}{l}\text { It is a hybrid analytical tool which includes R statistical and machine } \\
\text { learning algorithms }\end{array}$ \\
\hline
\end{tabular}

\section{MOTIVATION}

The financial sector is one of the first industries to face the Big Data challenges. Share market is one place where the time is money, cannot afford delay in processing. As the size of the data is growing the traditional systems are becoming obsolete and also cannot process unstructured data. The Hadoop could solve the aforementioned problems in an efficient way. 


\section{CONCLUSION}

This paper reviewed different studies on the data analytics with data mining approaches (algorithms and frameworks) and big data approaches, from the perception of data analysis with data mining problem this paper gives brief introduction to unstructured data and mining algorithms which consist of clustering, classification, and frequent patterns. From the perception of data analysis with big data this paper gives several studies which include metaheuristic algorithms, machine learning algorithms and statistical approaches. To process large volume of unstructured data efficient computational platforms or framework required so in this paper discussed different big data analysis frameworks. In future work implementing new mechanisms to process and analyze unstructured data efficiently.

\section{REFERENCES}

[1] "More CMOs Feeling Unprepared For the 'Data Explosion." Marketing Charts, 4 July, www.marketingcharts.com/digital-37207. 2017.

[2] Katal, Avita, et al. "Big Data: Issues, Challenges, Tools and Good Practices." 2013 Sixth International Conference on Contemporary Computing (IC3), 2013, doi:10.1109/ic3.2013.6612229.

[3] Sivarajah, Uthayasankar, et al. "Critical Analysis of Big Data Challenges and Analytical Methods." Journal of Business Research, vol. 70, 2017, pp. 263-286, DOI: 10.1016/j.jbusres.2016.08.001.

[4] Ding C, He X. K-means clustering via principal component analysis. In: Proceedings of the Twenty-first International Conference on Machine Learning, 2004, pp 1-9.

[5] Kollios G et.al, "Efficient biased sampling for approximate clustering and outlier detection in large data sets", Volume 15 issue 5 pp: 1170-87. 2003.

[6] Fisher D et.al, "Interactions with big data analytics. Interactions", volume 19 issue $3 \mathrm{pp:}$ 50-9. 2012.

[7] Laney D. 3D data management: controlling data volume, velocity, and variety, META Group, Tech. Rep. 2001.

Available:http://blogs.gartner.com/douglaney/files/2012/01/ad949-3D-Data-ManagementControlling-Data-Volume-Velocity-and-Variety.pdf.

[8] Van Rijmenam M, "Why the 3v's are not sufficient to describe big data". 2013.

[9] Borne K, "Top 10 big data challenges a serious look at 10 big data v's", Tech. Rep. 2014.

[10] Press G. \$16.1 billion big data market: 2014 predictions from IDC and IIA, Forbes, Tech. Rep. 2013. [Online]. Available: http://www.forbes.com/sites/gilpress/2013/12/12/16-1billion-big-data-market-2014-predictions-from-idc-and-iia/.

[11] Big data and analytics - an IDC four pillar research area, IDC, Tech. Rep. 2013. [Online]. Available: http://www.idc.com/prodserv/FourPillars/bigData/index.jsp.

[12] Taft DK. Big data market to reach \$46.34 billion by 2018, EWEEK, Tech. Rep. 2013. [Online]. Available: http://www.eweek.com/database/big-data-market-to-reach-46.34billion-by-2018.html.

[13] Research A. Big data spending to reach $\$ 114$ billion in 2018; look for machine learning to drive analytics, ABI Research, Tech. Rep. 2013. [Online]. Available: https://www.abiresearch.com/press/big-data-spending-to-reach-114-billion-in-2018-loo.

[14] Furrier J. Big data market $\$ 50$ billion by $2017-\mathrm{HP}$ vertica comes out \#1-according to wikibon research, SiliconANGLE, Tech. Rep. 2012. [Online]. Available: $\mathrm{http} / /$ siliconangle.com/blog/2012/02/15/big-data-market-15-billion-by-2017-hp-verticacomes-out-1-according-to-wikibon-research/. 
[15] Kelly J, Vellante D, Floyer D. Big data market size and vendor revenues, Wikibon, Tech. Rep.2014.[Online].Available:http://wikibon.org/wiki/v/Big_Data_Market_Size_and_Ven dor_Revenues.

[16] Kelly J, Floyer D, Vellante D, Miniman S. Big data vendor revenue and market forecast 2017,Wikibon,Tech.Rep.2014.[Online].Available:http://wikibon.org/wiki/v/Big_Data_Ve ndor_Revenue_and_Market_Forecast_2012-2017.

[17] Mayer-Schonberger V, Cukier K. Big data: a revolution that will transform how we live, work, and think. Boston: Houghton Mifflin Harcourt; 2013.

[18] Chen H, Chiang RHL, Storey VC. Business intelligence and analytics: from big data to big impact. MIS Quart. 2012;36(4):1165-88.

[19] Prasadu Peddi, Design of Simulators for Job Group Resource Allocation Scheduling in Grid and Cloud Computing Environments, ISSN: 2319-8753 volume 6 issue 8 pp: 1780517811. 2017.

[20] Kitchin R. The real-time city? big data and smart urbanism. Geo J. 2014;79(1):1-14.

[21] Fayyad UM, Piatetsky-Shapiro G, Smyth P. From data mining to knowledge discovery in databases. AI Mag. 17(3):37-54. 1996

\section{ABOUT AUTHOR}

Aarepu Lakshman Currently working as Assistant Professor in Malla Reddy Engineering College (Autonomous) Hyderabad, and he has 5 years of teaching experience, interested domains are Data Mining, Cloud computing, Machine Learning, Software Engineering and Big Data and He his published papers in national and international journals. 